\title{
Checkpoint Inhibition in the Treatment of Unresectable, Advanced Lymphoepithelioma-like Hepatocellular Carcinoma
}

\author{
David J. Hermel ${ }^{* 1}$, Emma Z. Du², Ray Lin ${ }^{3}$, Catherine T. Frenette 4 \\ and Darren S. Sigal ${ }^{1}$ \\ ${ }^{1}$ Division of Hematology and Oncology, Scripps Clinic and Scripps MD Anderson Cancer Center, La Jolla, CA, USA; \\ ${ }^{2}$ Division of Pathology, Scripps Clinic and Scripps MD Anderson Cancer Center, La Jolla, CA, USA; ${ }^{3}$ Division of Radiation On- \\ cology, Scripps Clinic and Scripps MD Anderson Cancer Center, La Jolla, CA, USA; ${ }^{4}$ Division of Gastroenterology,
} Scripps Clinic and Scripps MD Anderson Cancer Center, La Jolla, CA, USA

\begin{abstract}
Lymphoepithelioma-like hepatocellular carcinoma (LEL$\mathrm{HCC}$ ) is a very rare neoplasm, with distinct epidemiologic, morphologic and clinical characteristics. Molecular mechanistic insight into the pathogenesis of this carcinoma suggests a pivotal role for the host immune system in the proliferation and progression of this tumor. However, while detailed genomic profiling of these hepatic tumors have revealed an intra-tumoral inflammatory mutational signature that may predispose to immune checkpoint inhibitor efficacy, no published report has described their use in this tumor type. Unfortunately, with near 100 cases of LEL-HCC reported in the literature to date and the majority of cases confined to localized and resectable disease, current evidencebased practices in the unresectable setting are lacking, with unknown benefit of chemotherapy or immunotherapy. We report on the case of a 68 year-old man with unresectable, advanced LEL-HCC who had evidence of disease stability after starting on the immune checkpoint inhibitor nivolumab. His disease response persisted off therapy for over a year and was potentially augmented by radiotherapy at the site of local progression. For this extremely rare tumor subtype, this case highlights the potential efficacy and safety of immune checkpoint blockade in LEL-HCC and reinforces the need for more robust, large-scale analysis of patients with these rare tumors to better evaluate treatment strategies and outcomes.
\end{abstract}

Citation of this article: Hermel DJ, Du EZ, Lin R, Frenette $\mathrm{CT}$, Sigal DS. Checkpoint inhibition in the treatment of unresectable, advanced lymphoepithelioma-like hepatocellular carcinoma. J Clin Transl Hepatol 2021;000(000):000-000. doi: $10.14218 /$ JCTH.2020.00094.

Keywords: Immune checkpoint inhibitor; Lymphoepithelioma-like hepatocelluKeywords: Immune checkpoint inhibitor; Lymphoepithelioma-like hepatocelluAbbreviations: HCC, hepatocellular carcinoma; HCV hepatitis C virus; ICI immune checkpoint inhibitor; LEL-HCC, lymphoepithelioma-like hepatocellular carcinoma; LELC, lymphoepithelial-like carcinomas; MELD, model for end-stage liver disease; PD-1, programmed death-1; PDL-1, programmed death ligand 1; SBRT, stereotactic body radiation therapy; TACE, trans-arterial chemoembolization.

Received: 19 October 2020; Revised: 13 December 2020; Accepted: 05 January 2021

*Correspondence to: David J. Hermel, Division of Hematology and Oncology, Scripps Clinic, 10666 N Torrey Pines Rd, MS 312, La Jolla, CA 92037, USA Tel: +1-858-537-7617, Fax: +1-858-554-9100, E-mail: Hermel.david@scrippshealth.org

\section{Introduction}

Lymphoepithelioma-like hepatocellular carcinoma (LEL$\mathrm{HCC}$ ) is a rare hepatic neoplasm that is histologically characterized by a prominent reactive lymphoid infiltrate interspersed among undifferentiated carcinoma cells. ${ }^{1}$ Tumors with similar histopathologic features have been described in a variety of anatomical regions, yet primary liver involvement is an extremely rare entity that is traditionally classified into cholangiocarcinoma and HCC variants with unique etiological and clinical features. ${ }^{2}$ While the majority of reported cases of LEL-HCC tend to be early-stage, associated with preceding viral hepatitis and amenable to surgical resection, metastatic unresectable disease is seldom described and lacks clear evidence-based treatment guidelines. ${ }^{3}$ Incorporation of immune checkpoint inhibitors (ICIs), specifically agents that block inhibitory immune signaling through disruption of the interaction between programmed death-1 (PD-1) and its ligand (PD-L1), into the treatment paradigm of this disease has not been previously described. However, its use in this lymphocyte-enriched tumor type has a strong rationale for efficacy given the welldescribed correlation between ICI response and intra-tumoral lymphocyte concentration. ${ }^{4}$ Additionally, these novel agents have shown durable efficacy in a range of tumor types, with a safe toxicity profile. ${ }^{5}$

We report on a case of a patient with unresectable primary LEL-HCC who had a potential protracted and durable response to the PD-1 inhibitor nivolumab. Experiencing minimal toxicity, the patient had stable disease on nivolumab for approximately 3 years, with an isolated area of progression for which he received localized radiotherapy with appropriate tumor shrinkage. He completed a total of 63 doses of nivolumab and has continued to have stable disease off all therapy for more than 1 year. Our patient's clinical response demonstrates the potential efficacy and tolerability of single agent ICIs in this lymphocyte-enriched tumor subtype and suggests the potential benefit of localized radiotherapy to supplement ICI response in localized progression.

\section{Case report}

A 68 year-old man with a prior history of cirrhosis secondary to hepatitis $\mathrm{C}$ virus (HCV) presented to our clinic with a new enhancing $2.4 \mathrm{~cm}$ mass in segment 8 of the liver that was detected on abdominal magnetic resonance imaging. The decision was made to pursue trans-arterial chemoem- 

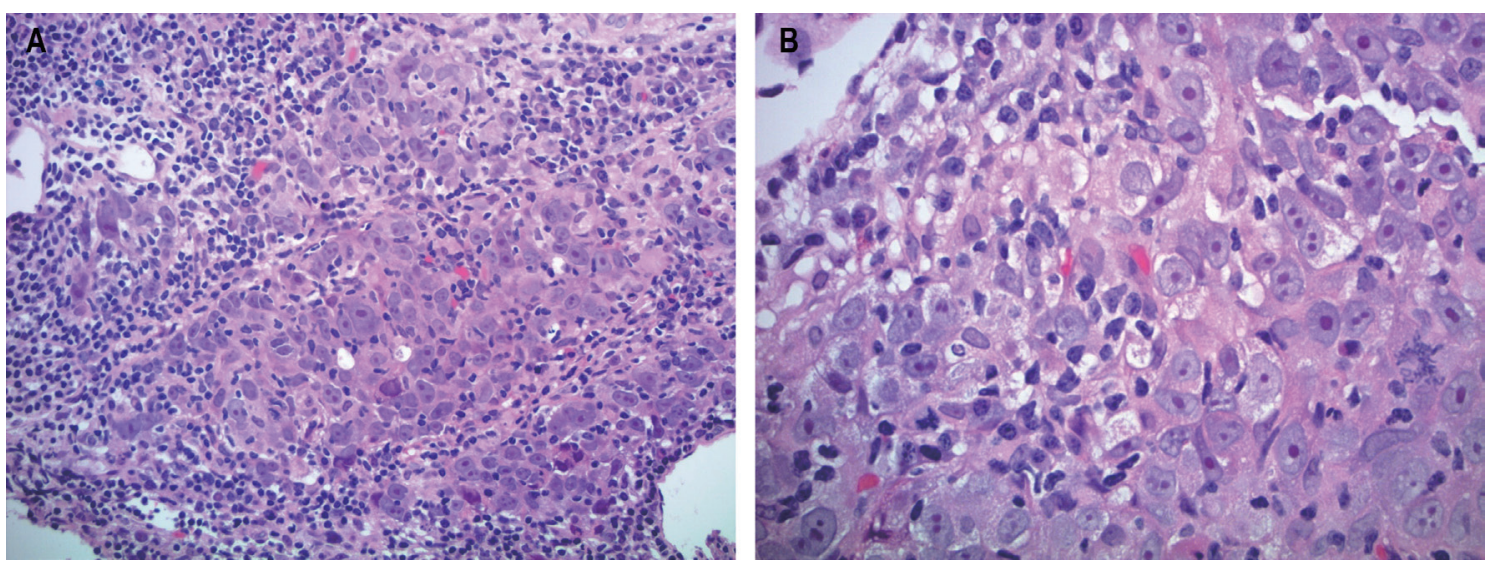

Fig. 1. Hematoxylin-eosin stain of the patient's tumor specimen. The morphology depicts clusters of large malignant cells with prominent nucleoli and eosinophilic cytoplasm with prominent infiltrating lymphocytes, viewed through (A) low and (B) high power.

bolization (referred to as TACE) of the lesion given tumor accessibility and his overall well-compensated, Child-Pugh A, model for end-stage liver disease (commonly known as MELD) 6 cirrhosis without associated portal hypertension. Despite his good initial radiographic response to TACE, follow-up imaging 2 months later revealed evidence of new periesophageal, gastrohepatic, and periportal lymphadenopathy.

He underwent a core needle biopsy of an enlarged subhepatic lymph node, which showed histologic evidence of LELHCC. Morphology was notable for large cells with abundant eosinophilic cytoplasm and prominent nucleoli associated with frequent lymphocytes (Fig. 1). Tumor cells expressed glutamine synthetase and CD10 by immunohistochemistry. Further immunostaining examinations showed negativity for CK7, CK19, CK20, and CK5 and positivity for the epithelial marker OSCAR (Fig. 2). The tumor tissue showed no evidence of microsatellite instability by polymerase chain reaction, and staining with the anti-PD-L1 clone SP142 showed PD-L1 expression in neoplastic cells at a tumor proportional score of $10 \%$ (Fig. 3). Serum alpha-fetoprotein level was 4 $\mathrm{ng} / \mathrm{mL}$. Due to the relatively rapid tumor progression, large tumoral lymphoid component and known efficacy data in HCC, the patient was initially started on a chemotherapy

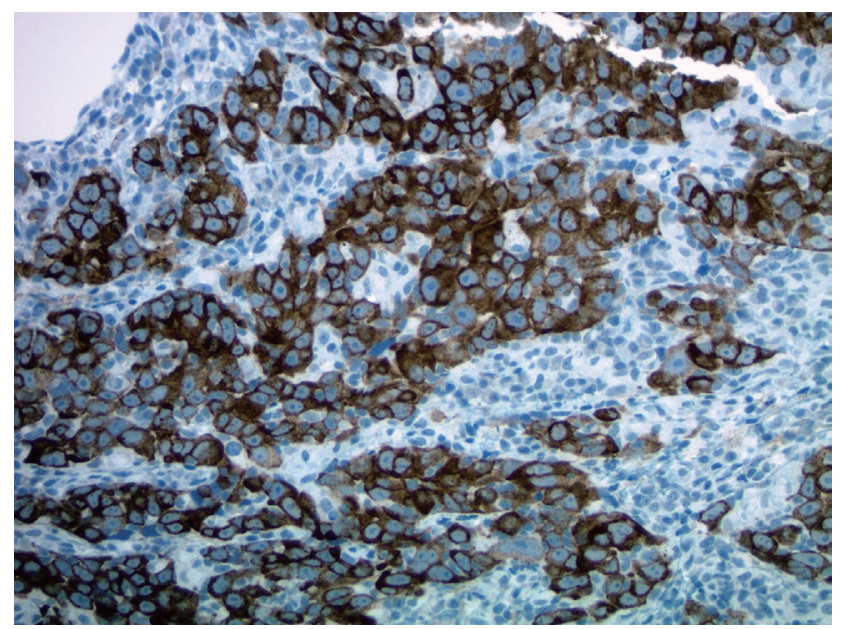

Fig. 2. Tumor stained with the anti-cytokeratin clone OSCAR. This epithelial marker is strongly positive in the tumor cells and supports the diagnosis of LEL-HCC. regimen of fluorouracil, folinic acid and oxaliplatin and underwent 7 cycles of therapy with stable disease but ongoing neuropathy limiting chemotherapy tolerance. Concomitantly, the patient underwent treatment for relapsed HCV with a 12-week course of sofosbuvir and velpatasvir and achieved a sustained virologic response.

He was then switched to single-agent nivolumab at 240 mg every 2 weeks and had stable porta hepatis lymphadenopathy for approximately 2 years on this ongoing therapy. His periesophageal, intra-abdominal and mediastinal lymphadenopathy regressed while on the therapy. The drug was well-tolerated, with development of a lichenoidrash that improved with topical treatment. After 2 years of relatively stable disease, he had evidence of subsequent progression in the porta hepatic lymph nodes and received palliative stereotactic body radiotherapy (referred to as SBRT) with significant localized regression on follow-up imaging. He continued nivolumab for 1 more year, which was ultimately discontinued due to patient preference and lack of clear evidence from early clinical trials that ICI treatment over 2 years improves overall survival. ${ }^{6}$ See Fig. 4 for complete treatment sequencing and relevant corresponding radiographic changes throughout treatment. To date, surveillance imaging continues to show stable dis-

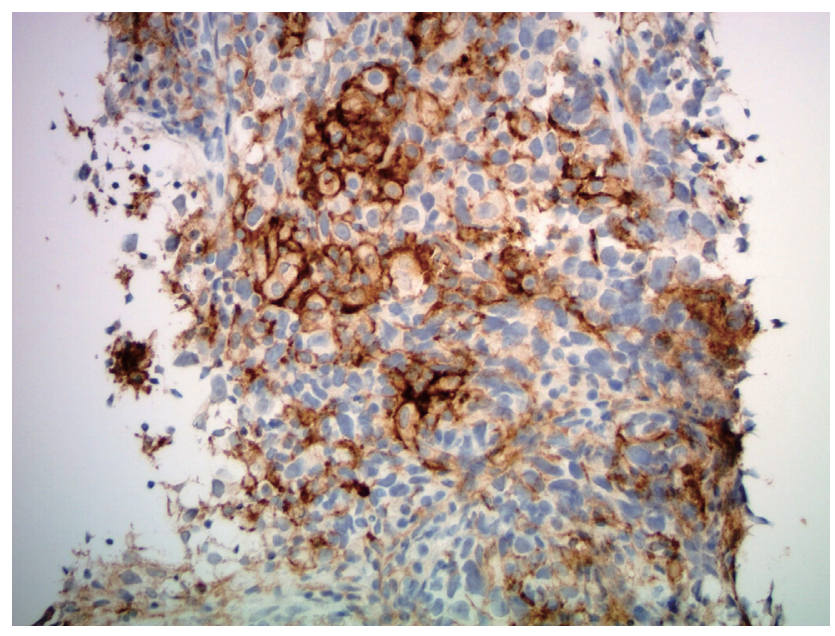

Fig. 3. Tumor tissue stained with the anti-PD-L1 clone SP142. The staining shows low level of PD-L1 expression, with tumor proportional score $(10 \%$, $2+)$. 
A

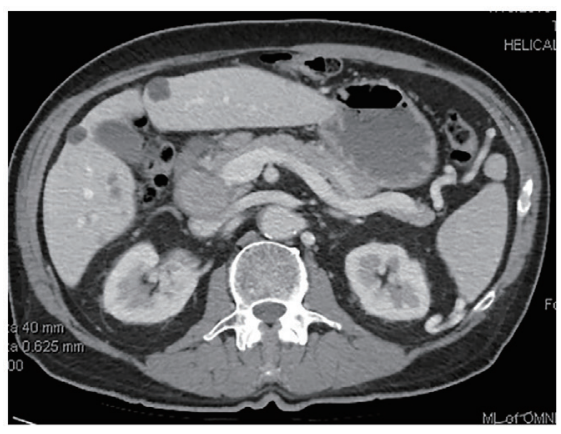

B

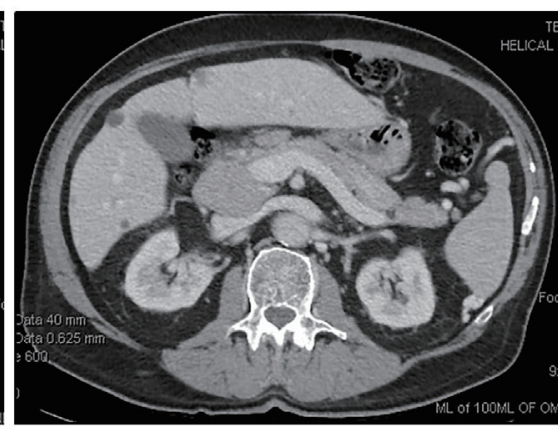

c

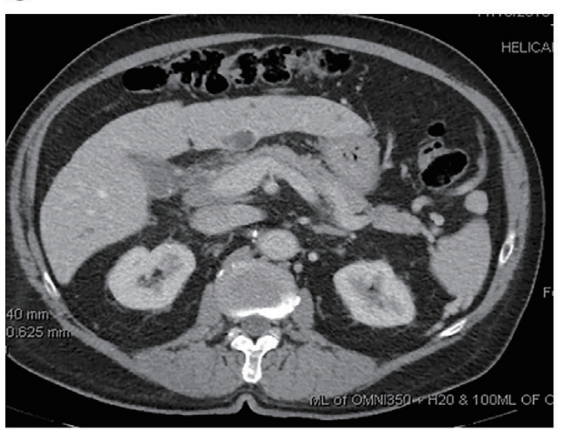

D

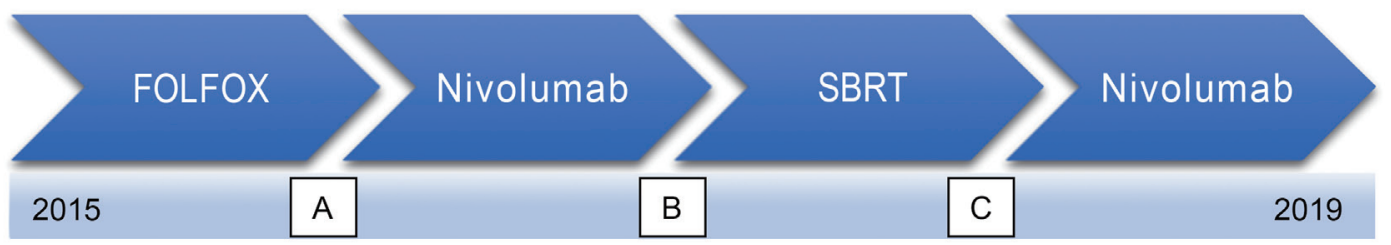

Fig. 4. Timeline of treatment course and corresponding radiographic findings. The following is a timeline of the different treatment modalities from the initiation of chemotherapy in 2015 to completion of nivolumab in the mid-2019s. At corresponding treatment points, there are computed tomography images showing changes in the patient's porta hepatic lymphadenopathy. These time points occur prior to initiation of nivolumab therapy (A), prior to initiation of SBRT (B), and after completion of SBRT (C). In each intervening computed tomography scan, the conglomerate porta hepatis lymph nodes change from $6.8 \mathrm{~cm} \times 4.6 \mathrm{~cm}$ to $7.7 \mathrm{~cm} \times 5.9$ $\mathrm{cm}$ to $3.7 \mathrm{~cm} \times 2.9 \mathrm{~cm}$, respectively.

ease without new or progressive disease over 1 year from his last dose of nivolumab and over 2 years since completion of SBRT.

\section{Discussion}

LEL-HCC represents a unique and rare subtype of HCC, with limited clinical evidence to guide systemic treatment options in advanced unresectable disease. The use of ICIs in this tumor type has not previously been described, though its potential efficacy has been previously postulated based on the distinct pathologic features of this tumor that are known to promote an immune-sensitizing tumoral milieu as well as potentiate ICI response in other tumor types. We describe a case of a patient with LEL-HCC who had durable disease control on ICI therapy, with stable disease persisting for over 4 years since ICI initiation and continuing off this therapy for over a year. The clinical course of his disease also demonstrates the potential utility of radiotherapy at local progression as a useful modality to supplement ICI treatment in LEL-HCC.

As an entity first described in 1995, LEL-HCC is defined by its histologic findings of undifferentiated large epithelial cells with prominent nucleoli that commonly stain positive for pankeratin (i.e. AE1/AE3) and hepatocyte specific antigen (i.e. HepPar1) and are intermixed with abundant lymphocytes primarily comprised of CD4 and CD8 T cells.7,8 Clinically, patients with LEL-HCC have been thought to have a more favorable prognosis than those with HCC, though our understanding of this disease is limited by its rarity and lack of published data. In the largest analysis of known cases, the epidemiologic, demographic, clinical characteristics and outcomes of 66 patients with LEL-HCC were evaluated. ${ }^{8}$ In this retrospective review of published cases, the median age at diagnosis was 58 years and the majority of patients were male $(64 \%)$ and white $(65 \%)$. Likewise, half of the patients with LEL-HCC had liver cirrhosis, $40 \%$ had hepatitis
$\mathrm{B}$ virus infection, and $34 \%$ had HCV infection. Most patients had very early stage disease, with $88 \%$ having a single focal lesion (the median size of which was $38 \mathrm{~mm}$ ), and consequently $91 \%$ of patients underwent surgical resection, with the remaining undergoing orthotopic liver transplantation. Though outcome data in this study are not available for all patients, review of individual retrospective case-series included in this study suggests a relatively low recurrence rate after surgery (of less than $9.1 \%$ ) and 5 -year survival rate of $94.1 \%$ to $100 \% .9,10$

While LEL-HCC is associated with a relatively favorable prognosis when diagnosed at early stages, there is a lack of clinical evidence guiding the disease course or treatment strategies in the advanced, non-operable setting. In the surgically operable setting, attempts have been made to incorporate post-operative chemotherapy; however, the benefit of this therapy is unclear in the adjuvant setting and cannot be reliably extrapolated to the metastatic setting. ${ }^{11-13}$ Nonetheless, chemotherapy has been proven to be efficacious in lymphoepithelial-like carcinomas (LELCs) of other anatomic locations. For example, a patient with LELC of the breast had a profound response to docetaxel, doxorubicin, and cyclophosphamide and incorporation of chemotherapy with surgery and radiation has yielded complete, durable responses in head and neck LELC patients. ${ }^{14,15}$

The use of ICIs in the treatment paradigm of LEL-HCC has not been previously described. One case report describes a 37 year-old woman with pulmonary LELC who was treated with nivolumab after progressive disease on systemic chemotherapy. ${ }^{16}$ Unfortunately, she died from complications shortly after starting nivolumab, so the effectiveness of this therapy is difficult to discern. Despite the paucity of clinical evidence, there are biologically plausible reasons to suggest potential efficacy of ICIs in LEL-HCC. Unlike conventional HCC, LEL-HCC has an increased intratumoral proportion of predominantly cytotoxic lymphocytes, which have been predictive of ICI response in various tumor subtypes. Likewise, although PD-L1 tumor expression has not been predictive of benefit of ICIs in HCC, there is 
evidence of elevated expression in LELCs, as in our patient, which may underlie potential ICI effectiveness as in other specific tumor subtypes. ${ }^{17}$

In addition to the above histologic findings that could suggest plausible benefit from ICIs in LEL-HCC, genomic comparisons between LEL-HCC and HCC have revealed mutational differences that suggest LEL-HCC may be more immunogenic and therefore potentially more susceptible to ICIs. Whole exome sequencing comparisons between conventional HCC and LEL-HCC have identified decreased overall nucleotide variants in specific genes of the Wnt/ $\beta$-catenin and Notch signaling pathways as well as focal amplification of chromosome $11 \mathrm{q} 13.3$ in LEL-HCC. ${ }^{18}$ The Wnt/ $\beta$-catenin and Notch signaling pathways are mechanistically important in driving an immunosuppressive tumoral milieu, and chromosome $11 \mathrm{q} 13.3$ is strongly associated with an immune checkpoint efficacy signature. 19,20 These somatic alterations in LEL-HCC suggest the potential benefit of immunotherapy. Together, the histopathologic, molecular and genomic features of LEL-HCC suggest a strong rationale for applicability and feasibility of ICIs in this tumor type.

\section{Conclusions}

We describe a patient with unresectable, advanced LEL-HCC who had evidence of sustained disease stability after starting nivolumab monotherapy. This case highlights a novel, previously undescribed treatment approach for patients with LEL-HCC in whom no supporting treatment data exist in the inoperable and non-transplant setting. The patient's durable disease control on ICI therapy in the context of the distinctive immunogenic pathologic features of this tumor suggest that there may be a broader role for ICI therapy in patients with LEL-HCC. However, as with any single case report in a rare, poorly-understood disease, it is difficult to definitely distinguish between causative treatment effect or the clinical spectrum of disease phenotype. Going forward, a collaborative, multi-institutional registry database is needed for patients with LEL-HCC to better understand the clinical course of this extremely rare hepatic neoplasm as well as the uptake and effectiveness of potential treatments.

\section{Funding}

None to declare.

\section{Conflict of interest}

The authors have no conflict of interests related to this publication.

\section{Author contributions}

Concept (DDS), data collection (DJH, DDS), drafting and writing of the manuscript $(\mathrm{DJH})$, critical revision of the manuscript for important intellectual content (EZD, RL, CTF, DSS).

\section{References}

1] Solinas A, Calvisi DF. Lessons from rare tumors: hepatic lymphoepitheliomalike carcinomas. World J Gastroenterol 2015;21:3472-3479. doi:10.3748/ wjg.v21.i12.3472.

[2] Zhang K, Tao C, Tao Z, Wu F, An S, Wu J, et al. Lymphoepithelioma-like carcinoma in liver not associated with Epstein-Barr virus: a report of 3 cases and literature review. Diagn Pathol 2020;15:115. doi:10.1186/s13000$020-01035-6$.

[3] Wang JK, Jin YW, Hu HJ, Regmi P, Ma WJ, Yang Q, et al. Lymphoepithelioma-like hepatocellular carcinoma: A case report and brief review of literature. Medicine (Baltimore) 2017;96:e9416. doi:10.1097/MD.000000 0000009416

[4] Paijens ST, Vledder A, de Bruyn M, Nijman HW. Tumor-infiltrating lymphocytes in the immunotherapy era. Cell Mol Immunol 2020. doi:10.1038/ s41423-020-00565-9.

[5] Hargadon $\mathrm{KM}$, Johnson $\mathrm{CE}$, Williams CJ. Immune checkpoint blockade therapy for cancer: An overview of FDA-approved immune checkpoint inhibitors. Int Immunopharmacol 2018:62:29-39. doi:10.1016/j.intimp. 2018.06.001.

[6] Topalian SL, Hodi FS, Brahmer JR, Gettinger SN, Smith DC, McDermott $\mathrm{DF}$, et al. Five-year survival and correlates among patients with advanced melanoma, renal cell carcinoma, or non-small cell lung cancer treated with nivolumab. JAMA Oncol 2019;5:1411-1420. doi:10.1001/jamaoncol. 2019.2187.

[7] Filotico M, Moretti V, Floccari F, D'Amuri A. Very rare liver neoplasm: Lymphoepithelioma-like (LEL) hepatocellular carcinoma. Case Rep Pathol 2018:2018:2651716. doi:10.1155/2018/2651716.

[8] Labgaa I, Stueck A, Ward SC. Lymphoepithelioma-Like carcinoma in liver. Am J Pathol 2017;187:1438-1444. doi:10.1016/j.ajpath.2017.02.022.

[9] Wada Y, Nakashima O, Kutami R, Yamamoto O, Kojiro M. Clinicopathological study on hepatocellular carcinoma with lymphocytic infiltration. Hepatology 1998;27:407-414. doi:10.1002/hep.510270214.

[10] Patel KR, Liu TC, Vaccharajani N, Chapman WC, Brunt EM. Characterization of inflammatory (lymphoepithelioma-like) hepatocellular carcinoma: study of 8 cases. Arch Pathol Lab Med 2014;138:1193-1202. doi:10.5858/ arpa.2013-0371-OA.

[11] Shinoda M, Kadota $Y$, Tsujikawa $H$, Masugi $Y$, Itano $O$, Ueno A, et al. Lymphoepithelioma-like hepatocellular carcinoma: a case report and a review of the literature. World J Surg Oncol 2013;11:97. doi:10.1186/1477-781911-97

12] Chen CJ, Jeng LB, Huang SF. Lymphoepithelioma-like hepatocellular carcinoma. Chang Gung Med J 2007;30:172-177.

[13] Chan AW, Tong JH, Pan Y, Chan SL, Wong GL, Wong VW, et al. Lymphoepithelioma-like hepatocellular carcinoma: an uncommon variant of hepatocellular carcinoma with favorable outcome. Am J Surg Pathol 2015:39: 304-312. doi:10.1097/PAS.0000000000000376.

[14] Nankin NL, Gondusky CJ, Abasolo PA, Kalantari BN. Lymphoepitheliomalike carcinoma of the breast. Radiol Case Rep 2015;10:963. doi:10.2484/ rcr.v10i1.963.

[15] Wenig BM. Lymphoepithelial-like carcinomas of the head and neck. Semin Diagn Pathol 2015;32:74-86. doi:10.1053/j.semdp.2014.12.004.

[16] Kim C, Rajan A, DeBrito PA, Giaccone G. Metastatic lymphoepithelioma-like carcinoma of the lung treated with nivolumab: a case report and focused review of literature. Transl Lung Cancer Res 2016;5:720-726. doi:10.21037/ tlcr.2016.11.06.

[17] Suster D, Pihan G, Mackinnon AC, Suster S. Expression of PD-L1/PD-1 in lymphoepithelioma-like carcinoma of the thymus. Mod Pathol 2018;31: 1801-1806. doi: 10.1038/s41379-018-0097-4

[18] Chan AW, Zhang Z, Chong CC, Tin EK, Chow C, Wong N. Genomic landscape of Iymphoepithelioma-like hepatocellular carcinoma. J Pathol 2019;249: 166-172. doi:10.1002/path.5313.

[19] Haseeb M, Pirzada RH, Ain QU, Choi S. Wnt signaling in the regulation of immune cell and cancer therapeutics. Cells 2019;8:1380. doi:10.3390/ cells 8111380

[20] Wang $F$, Ren $C$, Zhao $Q, X u N$, Shen L, Dai G, et al. Association of frequent amplification of chromosome 11q13 in esophageal squamous cell cancer with clinical benefit to immune check point blockade. J Clin Oncol 2019;37:4036. doi:10.1200/jco.2019.37.15_suppl.4036. 